\title{
Convergence a.e. of spherical partial Fourier integrals on weighted spaces for radial functions: endpoint estimates
}

by

\author{
María J. Carro (Barcelona) and Elena Prestini (Roma)
}

\begin{abstract}
We prove some extrapolation results for operators bounded on radial $L^{p}$ functions with $p \in\left(p_{0}, p_{1}\right)$ and deduce some endpoint estimates. We apply our results to prove the almost everywhere convergence of the spherical partial Fourier integrals and to obtain estimates on maximal Bochner-Riesz type operators acting on radial functions in several weighted spaces.
\end{abstract}

1. Introduction. For maximal spherical partial Fourier integrals as well as for maximal Bochner-Riesz means long standing open problems still remain to be solved concerning boundedness properties for general $f \in L^{p}\left(\mathbb{R}^{n}\right)$ while a lot is known in case $f$ is radial. The situation is similar for other operators such as general maximal spherical operators and Kakeya maximal operators ([7], [18], [19], [14], [1], [15], [28], [29], [17], [22]). Setting

$$
L_{\text {rad }}^{p}(\mu)=\left\{f \in L^{p}(\mu): f \text { is radial }\right\}
$$

where $\mu$ is a sigma-finite measure in $\mathbb{R}^{n}$, we are interested in operators $T$ such that

$$
T: L_{\mathrm{rad}}^{p}(\mu) \rightarrow L^{p}(\mu)
$$

is bounded for every $p \in\left(p_{0}, p_{1}\right)$ and the operator norm satisfies

$$
\|T\|_{p} \leq \frac{C}{\left(p-p_{0}\right)^{\alpha_{0}}\left(p-p_{1}\right)^{\alpha_{1}}},
$$

with $C$ a constant independent of $p$. This is the case for many of the examples in the above literature.

To obtain information at the endpoints $p_{0}, p_{1}$, one is led naturally from inequalities of the kind (1.1) to the theory of extrapolation of operators due to Yano (see [31]), which says that a sublinear operator satisfying an $L^{p}$ estimate, with constant $(p-1)^{-m}$ as $p \rightarrow 1^{+}$, is bounded from $L(\log L)^{m}$ to

2000 Mathematics Subject Classification: 26D10, 42B10.

Key words and phrases: extrapolation theory, Hardy-Littlewood maximal operator, Carleson maximal operator, Muckenhoupt weights. 
$L^{1}+L^{\infty}$. More recently the work by Antonov [2] has spurred new research on endpoint estimates ([30], [11], [10], [9]) and has brought new ideas to this old theory.

In Section 2 we obtain endpoint results for operators bounded on $L_{\mathrm{rad}}^{p}(\mu)$ with $p \in\left(p_{0}, p_{1}\right)$ using extrapolation techniques. We deal separately with the right and the left endpoint since the spaces involved are different.

In Section 3 we present some applications. First we prove the almost everywhere convergence of the spherical partial Fourier integrals of radial functions $f$ in certain weighted spaces such as $L^{p_{j}}(\log L)^{\beta_{j}}(w)$, as well as in weighted Lorentz spaces $\Lambda_{w}^{1}\left(t^{1 / p_{0}-1}\left(1+\log \frac{1}{t}\right)^{\beta_{0}}\right)$ and $\Lambda_{w}^{1}\left(t^{1 / p_{1}-1}(1+\log t)^{\beta_{1}}\right)$ where $w$ is a radial weight satisfying an $A_{1}$ condition and $\beta_{j}$ are positive numbers, $j=0,1$. The case $w=1$ has been studied in [15] and [27]. In the case of weighted $L^{p}$ spaces a result can be found in [26]. In the process we estimate the norm of the maximal Carleson operator in weighted $L^{p}$ spaces for certain weights in the Muchkenhoupt class $A_{p}([24])$. Our second application deals with maximal Bochner-Riesz type operators acting on radial functions introduced in [19]. For $n$ odd we improve the main result of $[19]$ at the endpoints.

We shall denote by $C$ a constant depending possibly on $p$ but uniformly bounded for all $p \in\left[p_{0}, p_{1}\right]$. Also $C$ might not be the same in all instances. We write $A \lesssim B$ if there exists a universal constant $C$ such that $A \leq C B$, and $A \approx B$ if $A \lesssim B$ and $B \lesssim A$. We shall work in $\mathbb{R}^{n}, \mu$ will be a sigma-finite measure on $\mathbb{R}^{n}$, and $w$ will be a radial weight in $\mathbb{R}^{n}$. As usual, $g_{\mu}^{*}(t)=\inf \left\{s: \lambda_{g}^{\mu}(s) \leq t\right\}$ is the decreasing rearrangement of $g$, where $\lambda_{g}^{\mu}(y)=\mu\left\{x \in \mathbb{R}^{n}:|g(x)|>y\right\}$ is the distribution function of $g$ with respect to the measure $\mu$ and $g_{\mu}^{* *}(t)=t^{-1} \int_{0}^{t} g_{\mu}^{*}(s) d s$. When the measure $\mu$ is given by $w(x) d x$ we shall write $g_{w}^{*}$ and $\lambda_{g}^{w}$ instead of using the subscript $\mu$ (we refer the reader to [4] for further information about distribution functions and decreasing rearrangements). For a measurable set $E, \chi_{E}$ denotes the characteristic function of $E,|E|$ denotes the Lebesgue measure of $E$ and $w(E)=\int_{E} w(x) d x$.

We mention that with obvious changes everything could be done for operators $T: L_{\mathrm{rad}}^{p}(\mu) \rightarrow L^{p}(\nu)$, but for simplicity of notation we shall consider $\mu=\nu$.

2. Endpoint estimates. Let us first recall that given a weight $v$ in $\mathbb{R}^{+}$, the weighted Lorentz spaces $\Lambda_{\mu}^{1}(v)$ and $\Gamma_{u}^{1, \infty}(v)$ are defined to be the sets of measurable functions such that

$$
\|f\|_{\Lambda_{\mu}^{1}(v)}=\int_{0}^{\infty} f_{\mu}^{*}(t) v(t) d t<\infty
$$


and

$$
\|f\|_{\Gamma_{\mu}^{1, \infty}(w)}=\sup _{t>0} V(t) f_{\mu}^{* *}(t)<\infty
$$

respectively, with $V(t)=\int_{0}^{t} v(s) d s$.

Since in this section the measure $\mu$ is fixed, we shall write $\|f\|_{p}=$ $\|f\|_{L^{p}(\mu)}, f_{\mu}^{*}=f^{*}, \lambda_{f}=\lambda_{f}^{\mu}, \Lambda_{\mu}^{1}(v)=\Lambda^{1}(v)$ and so on.

2.1. Let us start with the left endpoint $p_{0}$; that is, we shall assume that $T$ is a sublinear operator such that

$$
\|T f\|_{L^{p}} \lesssim \frac{1}{\left(p-p_{0}\right)^{\alpha}}\|f\|_{L_{\mathrm{rad}}^{p}(\mu)}
$$

for every $p \in\left(p_{0}, p_{1}\right)$ with $\alpha>0$.

Our first extrapolation result is the following:

THEOREM 2.1. If $T$ satisfies (2.1) and $f$ is a radial function, then

$$
\sup _{t>0} \frac{t^{1 / p_{0}}(T f)^{* *}(t)}{\left(1+\log ^{+} t\right)^{\alpha}} \lesssim \int_{0}^{\infty} f^{*}(t) t^{1 / p_{0}}\left(1+\log +\frac{1}{t}\right)^{\alpha} \frac{d t}{t}
$$

that is, $T: \Lambda_{\mathrm{rad}}^{1}\left(v_{0}\right) \rightarrow \Gamma^{1, \infty}\left(v_{1}\right)$ is bounded with

$$
v_{0}(t)=t^{1 / p_{0}-1}\left(1+\log ^{+} \frac{1}{t}\right)^{\alpha} \quad \text { and } \quad v_{1}(t)=t^{1 / p_{0}-1}\left(1+\log ^{+} t\right)^{-\alpha} \text {. }
$$

REMARK 2.2. At this point, it is important to observe that, if $p_{0}>1$, the above endpoint estimate is "near" (except for the logarithmic factors) to the restricted weak type estimate for $T: L_{\mathrm{rad}}^{p_{0}, 1}(\mu) \rightarrow L^{p_{0}, \infty}(\mu)$. However, we have to mention that, under our hypothesis, we cannot expect to get the restricted weak type estimate, since there are examples of operators satisfying (2.1) for which the restricted weak type inequality is known to be false. For instance (see [28]), the classical spherical maximal function was observed by Bourgain to be of restricted weak type at the endpoint $p=d /(d-1)$ when $d \geq 3$ but it is known to fail to be of restricted weak type at $p=2$ when $d=2$. We thank the referee for pointing out to us this concrete example.

Proof of Theorem 2.1. If $\|g\|_{\infty} \leq 1$ and $g$ is radial then

$$
(T g)^{* *}(t) t^{1 / p} \leq\|T g\|_{p} \lesssim \frac{1}{\left(p-p_{0}\right)^{\alpha}}\|g\|_{p} \lesssim \frac{1}{\left(p-p_{0}\right)^{\alpha}}\|g\|_{1}^{1 / p}
$$

and hence

$$
(T g)^{* *}(t) \lesssim \frac{1}{\left(p-p_{0}\right)^{\alpha}}\left(\frac{\|g\|_{1}}{t}\right)^{1 / p}
$$

Taking the infimum over $p_{0}<p<p_{1}$, we get

$$
(T g)^{* *}(t) \lesssim\left(\frac{\|g\|_{1}}{t}\right)^{1 / p_{0}}\left(1+\log ^{+} \frac{t}{\|g\|_{1}}\right)^{\alpha}
$$


Let now $f$ be a general radial function and let us write $f=\sum_{i \in \mathbb{Z}} 2^{i} f_{i}$ where $f_{i}=\left(f / 2^{i}\right) \chi_{\left\{2^{i-1} \leq|f|<2^{i}\right\}}$ is also radial and $\left\|f_{i}\right\|_{\infty} \leq 1$. Hence,

$$
(T f)^{* *}(t) \leq \sum_{i \in \mathbb{Z}} 2^{i}\left(T f_{i}\right)^{* *}(t) \lesssim \sum_{i \in \mathbb{Z}} 2^{i}\left(\frac{\left\|f_{i}\right\|_{1}}{t}\right)^{1 / p_{0}}\left(1+\log ^{+} \frac{t}{\left\|f_{i}\right\|_{1}}\right)^{\alpha}
$$

and since $\left\|f_{i}\right\|_{1} \leq \lambda_{f}\left(2^{i-1}\right)$, we obtain

$$
\sup _{t>0} \frac{t^{1 / p_{0}}(T f)^{* *}(t)}{\left(1+\log ^{+} t\right)^{\alpha}} \lesssim \sum_{i \in \mathbb{Z}} 2^{i} D\left(\lambda_{f}\left(2^{i}\right)\right) \approx \int_{0}^{\infty} D\left(\lambda_{f}(y)\right) d y
$$

with $D(x)=x^{1 / p_{0}}\left(1+\log ^{+} \frac{1}{x}\right)^{\alpha}$. The result now follows since

$$
\int_{0}^{\infty} D\left(\lambda_{f}(y)\right) d y \approx \int_{0}^{\infty} f^{*}(t) t^{1 / p_{0}}\left(1+\log ^{+} \frac{1}{t}\right)^{\alpha} \frac{d t}{t}
$$

This result is the best known for the case $p_{0}=1$. However, if $p_{0}>1$, it can be improved using some technical results of interpolation theory plus a modification adapted to radial functions of some extrapolation techniques developed in [11].

Let us start with a technical lemma.

LEMmA 2.3. Let $G$ be a concave function such that

$$
G(t) \approx\left(\int_{0}^{t_{0}^{p_{0}}} g^{*}(s)^{p_{0}} d s\right)^{1 / p_{0}}
$$

and let, for every $i \in \mathbb{Z}$,

$$
E_{i}=\left\{s \in(0, \infty): G^{\prime}(s)>2^{i}\right\} .
$$

Then there exist $\left(g_{i}\right)_{i \in \mathbb{Z}}$ such that $g=\sum_{i} 2^{i} g_{i}$ and

$$
\left(\int_{0}^{t^{p_{0}}} g_{i}^{*}(s)^{p_{0}} d s\right)^{1 / p_{0}} \lesssim \min \left(t,\left|E_{i}\right|\right) .
$$

Proof. Let us first mention that since the function

$$
H(t)=\left(\int_{0}^{t^{p_{0}}} g^{*}(s)^{p_{0}} d s\right)^{1 / p_{0}}
$$

is increasing and $H(s) / s$ is decreasing, $H$ is quasi-concave and hence equivalent to a concave function; so the existence of $G$ is clear. Since $G^{\prime}$ is a decreasing function we have $G^{\prime}(s) \leq 4 \sum_{i} 2^{i} \chi_{E_{i}}(s)$ and $G(t) \lesssim \sum_{i} 2^{i} \min \left(t,\left|E_{i}\right|\right)$. On the other hand, $H(t) \approx K\left(g, t ; L^{p_{0}}, L^{\infty}\right)$ and thus we can use the Kdivisibility theorem of interpolation theory $([5, \mathrm{p} .325])$ to prove the result.

Our next theorem is an improvement of Theorem 2.1. 
THEOREM 2.4. If $T$ satisfies (2.1), then for every radial function $f$,

$$
\sup _{t>0} \frac{\left(\int_{0}^{t}\left[(T f)^{*}(s)\right]^{p_{0}} d s\right)^{1 / p_{0}}}{\left(1+\log ^{+} t\right)^{\alpha}} \lesssim\|f\|_{p_{0}}+\int_{0}^{1} \frac{\left(\int_{0}^{t} f^{*}(s)^{p_{0}} d s\right)^{1 / p_{0}}}{t}\left(\log \frac{1}{t}\right)^{\alpha-1} d t .
$$

Proof. Let $f$ be a radial function on $\mathbb{R}^{n}$ and let $g$ be defined in $(0, \infty)$ by $g(u)=f\left(u^{1 / n}\right)$. Then simple computations show that $f^{*}(t)=g^{*}\left(c_{n} t\right)$ for a certain constant $c_{n}$ depending only on $n$.

Given $g$ we can apply Lemma 2.3 to deduce that $g=\sum_{i \in \mathbb{Z}} 2^{i} g_{i}$, where

$$
\left(\int_{0}^{t_{0}} g_{i}^{*}(s)^{p_{0}} d s\right)^{1 / p_{0}} \lesssim \min \left(t,\left|E_{i}\right|\right) .
$$

Taking $f_{i}$ radial such that $f_{i}\left(u^{1 / n}\right)=g_{i}(u)$ we find that $f=\sum_{i \in \mathbb{Z}} 2^{i} f_{i}$ and

$$
\left(\int_{0}^{t^{p_{0}}} f_{i}^{*}(s)^{p_{0}} d s\right)^{1 / p_{0}} \lesssim \min \left(t,\left|E_{i}\right|\right)
$$

or equivalently

$$
\int_{0}^{t} f_{i}^{*}(s)^{p_{0}} d s \lesssim \min \left(t,\left|E_{i}\right|^{p_{0}}\right) .
$$

From this it follows (see $\left[4\right.$, p. 61]) that, for every $p \geq p_{0}$,

$$
\left\|f_{i}\right\|_{p} \lesssim\left|E_{i}\right|^{p_{0} / p}
$$

Then, for every $i \in \mathbb{Z}$ and every $p>p_{0}$,

$$
\begin{aligned}
\left(\frac{1}{t} \int_{0}^{t}\left[\left(T f_{i}\right)^{*}(s)\right]^{p_{0}} d s\right)^{1 / p_{0}} & \leq\left(\frac{1}{t} \int_{0}^{t}\left[\left(T f_{i}\right)^{*}(s)\right]^{p} d s\right)^{1 / p} \leq t^{-1 / p}\left\|T f_{i}\right\|_{p} \\
& \lesssim t^{-1 / p} \frac{1}{\left(p-p_{0}\right)^{\alpha}}\left\|f_{i}\right\|_{p} \leq \frac{1}{\left(p-p_{0}\right)^{\alpha}}\left(\frac{\left|E_{i}\right|^{p_{0}}}{t}\right)^{1 / p} .
\end{aligned}
$$

Taking the infimum over $p_{0}<p \leq q<p_{1}$ we obtain

$$
\left(\frac{1}{t} \int_{0}^{t}\left[\left(T f_{i}\right)^{*}(s)\right]^{p_{0}} d s\right)^{1 / p_{0}} \lesssim \frac{\left|E_{i}\right|}{t^{1 / p_{0}}}\left(1+\log +\frac{t^{1 / p_{0}}}{\left|E_{i}\right|}\right)^{\alpha}
$$

and therefore

$$
\sup _{t>0} \frac{\left(\int_{0}^{t}\left[\left(T f_{i}\right)^{*}(s)\right]^{p_{0}} d s\right)^{1 / p_{0}}}{\left(1+\log ^{+} t\right)^{\alpha}} \lesssim\left|E_{i}\right|\left(1+\log ^{+} \frac{1}{\left|E_{i}\right|}\right)^{\alpha}=: D\left(\left|E_{i}\right|\right) .
$$

Summing over $i \in \mathbb{Z}$ we obtain

$$
\sup _{t>0} \frac{\left(\int_{0}^{t}\left[(T f)^{*}(s)\right]^{p_{0}} d s\right)^{1 / p_{0}}}{\left(1+\log ^{+} t\right)^{\alpha}} \lesssim \sum_{i \in \mathbb{Z}} 2^{i} D\left(\left|E_{i}\right|\right) \approx \int_{0}^{\infty} D\left(\lambda_{G^{\prime}}(y)\right) d y,
$$


where $G$ is defined as in Lemma 2.3,

$$
\begin{aligned}
G(t) & \approx\left(\int_{0}^{t^{p_{0}}} g^{*}(s)^{p_{0}} d s\right)^{1 / p_{0}}=\left(\int_{0}^{t^{p_{0}}} f^{*}\left(\frac{s}{c_{n}}\right)^{p_{0}} d s\right)^{1 / p_{0}} \\
& =\left(c_{n} \int_{0}^{t^{p_{0}} / c_{n}} f^{*}(s)^{p_{0}} d s\right)^{1 / p_{0}} \approx\left(\int_{0}^{t_{0}} f^{*}(s)^{p_{0}} d s\right)^{1 / p_{0}} .
\end{aligned}
$$

Thus, integrating by parts gives

$$
\begin{aligned}
\sup _{t>0} \frac{\left(\int_{0}^{t}\left[(T f)^{*}(s)\right]^{p_{0}} d s\right)^{1 / p_{0}}}{\left(1+\log ^{+} t\right)^{\alpha}} \lesssim \int_{0}^{\infty} G^{\prime}(t) d D(t) \\
\quad=\int_{0}^{1} G^{\prime}(t)\left(\log \frac{1}{t}\right)^{\alpha} d t+\int_{1}^{\infty} G^{\prime}(t) d t \lesssim\|f\|_{p_{0}}+\int_{0}^{1} \frac{G(t)}{t}\left(\log \frac{1}{t}\right)^{\alpha-1} d t \\
\lesssim\|f\|_{p_{0}}+\int_{0}^{1} \frac{\left(\int_{0}^{t_{0}^{p_{0}}} f^{*}(s)^{p_{0}} d s\right)^{1 / p_{0}}}{t}\left(\log \frac{1}{t}\right)^{\alpha-1} d t
\end{aligned}
$$

as we wanted to prove.

Definition 2.5. We define the spaces $D_{p, \alpha}^{+}$and $R_{p, \alpha}^{+}$by the norms

$$
\|f\|_{D_{p, \alpha}^{+}}=\|f\|_{p}+\int_{0}^{1} \frac{\left(\int_{0}^{t} f^{*}(s)^{p} d s\right)^{1 / p}}{t}\left(\log \frac{1}{t}\right)^{\alpha-1} d t
$$

and

$$
\|f\|_{R_{p, \alpha}^{+}}=\sup _{t>0} \frac{\left(\int_{0}^{t} f^{*}(s)^{p} d s\right)^{1 / p}}{\left(1+\log ^{+} t\right)^{\alpha}}
$$

Also,

$$
D_{p, \alpha, \text { rad }}^{+}=\left\{f \in D_{p, \alpha}^{+}: f \text { is radial }\right\} .
$$

With these definitions, Theorem 2.4 can be stated as follows:

Theorem 2.6. If $T$ satisfies (2.1) then $T: D_{p_{0}, \alpha, \mathrm{rad}}^{+} \rightarrow R_{p_{0}, \alpha}^{+}$is bounded.

In the following remark, we compare our spaces above with classical Orlicz and Lorentz spaces.

REMARK 2.7. 1. Theorem 2.4 is an improvement of Theorem 2.1: to see this, let us just recall that $L^{p_{0}, 1} \subset L^{p_{0}}([4])$ and, in fact (see [13]),

$$
\sup _{f} \frac{\left(\int_{0}^{t} f^{*}(s)^{p_{0}} d s\right)^{1 / p_{0}}}{\int_{0}^{t} f^{*}(s) s^{1 / p_{0}-1} d t}=\sup _{r>0} \frac{\left(\int_{0}^{t} \chi_{(0, r)} d s\right)^{1 / p_{0}}}{\int_{0}^{t} \chi_{(0, r)} s^{1 / p_{0}-1} d t}=p_{0}<\infty .
$$


Hence

$$
\begin{aligned}
\|f\|_{D_{p_{0}, \alpha}^{+}} & =\|f\|_{p_{0}}+\int_{0}^{1} \frac{\left(\int_{0}^{t} f^{*}(s)^{p_{0}} d s\right)^{1 / p_{0}}}{t}\left(\log \frac{1}{t}\right)^{\alpha-1} d t \\
& \leq\|f\|_{p_{0}}+\int_{0}^{1} \frac{\int_{0}^{t} f^{*}(s) s^{1 / p_{0}-1} d s}{t}\left(\log \frac{1}{t}\right)^{\alpha-1} d t \\
& \lesssim \int_{0}^{\infty} f^{*}(s) s^{1 / p_{0}-1}\left(1+\log \frac{1}{s}\right)^{\alpha} d s=\|f\|_{\Lambda^{1}\left(v_{0}\right)},
\end{aligned}
$$

with $v_{0}$ as in the statement of Theorem 2.1. Therefore

$$
\Lambda^{1}\left(v_{0}\right) \subset D_{p_{0}, \alpha}^{+}
$$

In fact, the above inclusion is strict since if we take $f$ such that

$$
f^{*}(s)=s^{-1 / p_{0}}\left(1+\log ^{+} \frac{1}{s}\right)^{-\beta} \quad \text { with } 1 / p_{0}+\alpha<\beta<1+\alpha
$$

then we have $\|f\|_{\Lambda^{1}\left(v_{0}\right)}=\infty$ while $\|f\|_{D_{p_{0}, \alpha}^{+}}<\infty$.

Also, if $v_{1}$ is as in the statement of Theorem 2.1,

$$
\|f\|_{\Gamma^{1, \infty}\left(v_{1}\right)}=\sup _{t>0} \frac{t^{1 / p_{0}} f^{* *}(t)}{\left(1+\log ^{+} t\right)^{\alpha}} \leq \sup _{t>0} \frac{\left(\int_{0}^{t} f^{*}(s)^{p_{0}} d s\right)^{1 / p_{0}}}{\left(1+\log ^{+} t\right)^{\alpha}}=\|f\|_{R_{p_{0}, \alpha}^{+}}
$$

and hence

$$
R_{p_{0}, \alpha}^{+} \subset \Gamma^{1, \infty}\left(v_{1}\right) .
$$

Moreover, this inclusion is strict since taking $f$ such that $f^{*}(s)=s^{-1 / p_{0}}$ we have $\|f\|_{R_{p_{0}, \alpha}^{+}}=\infty$ while $\|f\|_{\Gamma^{1, \infty}\left(v_{1}\right)}<\infty$.

2. $D_{p_{0}, \alpha}^{+}$is not comparable with $L^{p_{0}, 1}$ for any $\alpha$. To see this, we observe first that taking

$$
f^{*}(t)=\chi_{(0,1)}(t)+\frac{1}{t^{1 / p_{0}}\left(\log \frac{1}{t}\right)} \chi_{(1, \infty)}(t)
$$

we have $f \in D_{p_{0}, \alpha}^{+} \backslash L^{p_{0}, 1}$. For the converse, we have to apply Theorem 4.1(ii) of [12] to deduce that, for every $\alpha>0$,

$$
\sup _{f} \frac{\int_{0}^{1}\left(\int_{0}^{t} f^{*}(s)^{p_{0}} d s\right)^{1 / p_{0}}\left(\log \frac{1}{t}\right)^{\alpha-1} \frac{d t}{t}}{\int_{0}^{1} f^{*}(s) s^{1 / p_{0}-1} d t}=\infty .
$$


3. If $\beta / p_{0}>\alpha$, then

$$
\begin{aligned}
\int_{0}^{1} \frac{\left(\int_{0}^{t_{0}} f^{*}(s)^{p_{0}} d s\right)^{1 / p_{0}}}{t}\left(\log \frac{1}{t}\right)^{\alpha-1} d t & \leq \int_{0}^{1} \frac{\left(\int_{0}^{t^{p_{0}}} f^{*}(s)^{p_{0}}\left(1+\log \frac{1}{s}\right)^{\beta} d s\right)^{1 / p_{0}}}{t\left(1+\log \frac{1}{t}\right)^{\beta / p_{0}+1-\alpha}} d t \\
& \leq\left(\int_{0}^{\infty} f^{*}(s)^{p_{0}}\left(1+\log \frac{1}{s}\right)^{\beta} d s\right)^{1 / p_{0}} \\
& \lesssim\|f\|_{L^{p_{0}}(\log L)^{\beta}},
\end{aligned}
$$

that is, $L^{p_{0}}(\log L)^{\beta} \subset D_{p_{0}, \alpha}^{+}$.

2.2. Now we consider the right endpoint $p_{1}$. Let $T$ be a sublinear operator such that, for every $p_{0} \leq p<p_{1}$,

$$
\|T f\|_{L^{p}(\mu)} \lesssim \frac{1}{\left(p_{1}-p\right)^{\alpha}}\|f\|_{L_{\mathrm{rad}}^{p}(\mu)} .
$$

TheOREm 2.8. If $T$ satisfies (2.2) then, for every radial function $f$,

$$
\sup _{t>0} \frac{t^{1 / p_{1}}(T f)^{* *}(t)}{\left(1+\log ^{+} \frac{1}{t}\right)^{\alpha}} \lesssim \int_{0}^{\infty} f^{*}(t) t^{1 / p_{1}}\left(1+\log ^{+} t\right)^{\alpha} \frac{d t}{t} ;
$$

that is, $T: \Lambda^{1}\left(v_{0}\right) \rightarrow \Gamma^{1, \infty}\left(v_{1}\right)$ is bounded with $v_{0}(t)=t^{1 / p_{1}-1}\left(1+\log ^{+} t\right)^{\alpha}$ and $v_{1}(t)=t^{1 / p_{1}-1}\left(1+\log ^{+} \frac{1}{t}\right)^{-\alpha}$.

Proof. Let $g$ be a radial function such that $\|g\|_{\infty} \leq 1$. Then

$$
(T g)^{* *}(t) \lesssim\left(\frac{1}{p_{1}-p}\right)^{\alpha}\left(\frac{\|g\|_{1}}{t}\right)^{1 / p}
$$

and taking the infimum over $p_{0} \leq p<p_{1}$ we obtain

$$
(T g)^{* *}(t) \lesssim\left(\frac{\|g\|_{1}}{t}\right)^{1 / p_{1}}\left(1+\log ^{+} \frac{\|g\|_{1}}{t}\right)^{\alpha} .
$$

Let now $f$ be a general radial function and let us write $f=\sum_{i \in \mathbb{Z}} 2^{i} f_{i}$ where $f_{i}=\left(f / 2^{i}\right) \chi_{\left\{2^{i-1} \leq|f|<2^{i}\right\}}$. As in Theorem 2.1 we obtain

$$
\sup _{t>0} \frac{t^{1 / p_{1}}(T f)^{* *}(t)}{\left(1+\log ^{+} \frac{1}{t}\right)^{\alpha}} \lesssim \int_{0}^{\infty} D\left(\lambda_{f}(y)\right) d y
$$

with $D(x)=x^{1 / p_{1}}\left(1+\log ^{+} x\right)^{\alpha}$. The result now follows since

$$
\int_{0}^{\infty} D\left(\lambda_{f}(y)\right) d y \approx \int_{0}^{\infty} f^{*}(t) t^{1 / p_{1}}\left(1+\log ^{+} t\right)^{\alpha} \frac{d t}{t} .
$$

To improve the above result in the case $p_{1}<\infty$, we have to proceed as in Theorem 2.4. 
LEMma 2.9. Let $G$ be a concave function such that

$$
G(t) \approx t\left(\int_{t^{p_{1}^{\prime}}}^{\infty} g^{* *}(s)^{p_{1}} d s\right)^{1 / p_{1}}
$$

and let, for every $i \in \mathbb{Z}$,

$$
E_{i}=\left\{s \in(0, \infty): G^{\prime}(s)>2^{i}\right\} .
$$

Then there exist $\left(g_{i}\right)_{i \in \mathbb{Z}}$ such that $g=\sum_{i} 2^{i} g_{i}$ and

$$
t\left(\int_{t^{p_{1}^{\prime}}}^{\infty} g_{i}^{* *}(s)^{p_{1}} d s\right)^{1 / p_{1}} \lesssim \min \left(t,\left|E_{i}\right|\right) .
$$

Proof. It suffices to observe that $G(t) \approx K\left(g, t ; L^{1}, L^{p_{1}}\right)$ (see [4]) and proceed as in Lemma 2.3.

Theorem 2.10. If $T$ satisfies (2.2) then, for every $f$ radial,

$$
\begin{aligned}
& \sup _{t>0} \frac{\left(\int_{t}^{\infty}(T f)^{* *}(s)^{p_{1}} d s\right)^{1 / p_{1}}}{\left(1+\log ^{+} \frac{1}{t}\right)^{\alpha}} \\
& \lesssim\|f\|_{p_{1}}+\int_{1}^{\infty} \frac{\left(\int_{t}^{\infty} f^{* *}(s)^{p_{1}} d s\right)^{1 / p_{1}}}{t}\left(1+\log ^{+} t\right)^{\alpha-1} d t .
\end{aligned}
$$

Proof. Let $f \in L_{\mathrm{rad}}^{p_{1}}\left(\mathbb{R}^{n}\right)$ be such that $\|f\|_{p_{1}}=c$ with $c$ to be chosen later, and let $g$ be defined in $(0, \infty)$ by $g(u)=f\left(u^{1 / n}\right)$.

Now given $g$ we can apply Lemma 2.9 to deduce that $g=\sum_{i \in \mathbb{Z}} 2^{i} g_{i}$, where

$$
t\left(\int_{t^{p_{1}^{\prime}}}^{\infty} g_{i}^{* *}(s)^{p_{1}} d s\right)^{1 / p_{1}} \lesssim \min \left(t,\left|E_{i}\right|\right) .
$$

with $E_{i}=\left\{s: G^{\prime}(s)>2^{i}\right\}$ and $G$ defined as in Lemma 2.9.

Since $G^{\prime}$ is decreasing,

$$
G^{\prime}(t) \leq \frac{1}{t} \int_{0}^{t} G^{\prime}(s) d s=\frac{G(t)}{t} \lesssim\|f\|_{p_{1}} \approx c,
$$

and hence choosing $c$ such that $G^{\prime}(t) \leq 1$ we find that $E_{i}=\emptyset$ whenever $i>0$. Taking $f_{i}$ radial such that $f_{i}\left(u^{1 / n}\right)=g_{i}(u)$ we have $f=\sum_{i=-\infty}^{0} 2^{i} f_{i}$ and

$$
t\left(\int_{t^{\prime}}^{\infty} f_{i}^{* *}(s)^{p_{1}} d s\right)^{1 / p_{1}} \lesssim \min \left(t,\left|E_{i}\right|\right) .
$$


From this it follows that $\left\|f_{i}\right\|_{p_{1}} \lesssim 1$ and since

$$
\begin{aligned}
\int_{0}^{t} f^{*}(s) d s & \approx t^{1 / p_{1}^{\prime}}\left(\int_{t}^{\infty} \frac{1}{s^{p_{1}}} d s\right)^{1 / p_{1}} \int_{0}^{t} f^{*}(s) d s \\
& \lesssim t^{1 / p_{1}^{\prime}}\left(\int_{t}^{\infty} \frac{\left(\int_{0}^{s} f^{*}(u) d u\right)^{p_{1}}}{s^{p_{1}}} d s\right)^{1 / p_{1}} \lesssim \min \left(t^{1 / p_{1}^{\prime}},\left|E_{i}\right|\right)
\end{aligned}
$$

we see that $\left\|f_{i}\right\|_{1} \lesssim\left|E_{i}\right|$ and hence, for every $p<p_{1}$,

$$
\left\|f_{i}\right\|_{p} \lesssim\left|E_{i}\right|^{1-p_{1}^{\prime}+p_{1}^{\prime} / p}
$$

Then, for every $i \in \mathbb{Z}^{-}$and every $p<p_{1}$,

$$
\begin{aligned}
\left(\int_{t^{p_{1}^{\prime}}}^{\infty}\left[\left(T f_{i}\right)^{* *}(s)\right]^{p_{1}} d s\right)^{1 / p_{1}} \lesssim\left(\int_{t^{p_{1}^{\prime}}}^{\infty}\left[\left(T f_{i}\right)^{* *}(s)\right]^{p} s^{p / p_{1}-1} d s\right)^{1 / p} \\
\lesssim t^{p_{1}^{\prime}\left(1 / p_{1}-1 / p\right)}\left\|T f_{i}\right\|_{p} \lesssim t^{p_{1}^{\prime}\left(1 / p_{1}-1 / p\right)}\left(\frac{1}{p_{1}-p}\right)^{\alpha}\left\|f_{i}\right\|_{p} \\
\leq\left(\frac{1}{p_{1}-p}\right)^{\alpha}\left(\frac{\left|E_{i}\right|}{t}\right)^{1-p_{1}^{\prime}}\left(\frac{\left|E_{i}\right|}{t}\right)^{p_{1}^{\prime} / p} .
\end{aligned}
$$

Taking the infimum over $p_{0} \leq p<p_{1}$ we obtain

$$
\left(\int_{t^{p_{1}^{\prime}}}^{\infty}\left[\left(T f_{i}\right)^{* *}(s)\right]^{p_{1}} d s\right)^{1 / p_{1}} \lesssim\left(1+\log ^{+} \frac{\left|E_{i}\right|}{t}\right)^{\alpha}
$$

and therefore

$$
\sup _{t>0} \frac{\left(\int_{t^{p_{1}}}^{\infty}\left[\left(T f_{i}\right)^{* *}(s)\right]^{p_{1}} d s\right)^{1 / p_{1}}}{\left(1+\log ^{+} \frac{1}{t}\right)^{\alpha}} \lesssim\left(1+\log ^{+}\left|E_{i}\right|\right)^{\alpha}=: D\left(\left|E_{i}\right|\right) .
$$

Summing over $i \in \mathbb{Z}^{-}$we obtain

$$
\begin{aligned}
\sup _{t>0} \frac{\left(\int_{t}^{\infty}\left[(T f)^{* *}(s)\right]^{p_{1}} d s\right)^{1 / p_{1}}}{\left(1+\log ^{+} \frac{1}{t}\right)^{\alpha}} & \lesssim \sum_{i \in \mathbb{Z}^{-}} 2^{i} D\left(\left|E_{i}\right|\right) \approx \int_{0}^{1} D\left(\lambda_{G^{\prime}}(y)\right) d y \\
& \approx 1+\int_{0}^{\infty}\left(\log ^{+}\left(\lambda_{G^{\prime}}(y)\right)\right)^{\alpha} d y
\end{aligned}
$$

Integrating by parts gives

$$
\begin{aligned}
& \sup _{t>0} \frac{\left(\int_{t}^{\infty}\left[(T f)^{* *}(s)\right]^{p_{1}} d s\right)^{1 / p_{1}}}{\left(1+\log ^{+} \frac{1}{t}\right)^{\alpha}} \lesssim 1+\int_{1}^{\infty} G^{\prime}(t)(\log t)^{\alpha-1} \frac{d t}{t} \\
& \lesssim 1+\int_{1}^{\infty} G(t)(\log t)^{\alpha-1} \frac{d t}{t^{2}} \leq 1+\int_{1}^{\infty} \frac{\left(\int_{t}^{\infty} f^{* *}(s)^{p_{1}} d s\right)^{1 / p_{1}}}{t}(\log t)^{\alpha-1} d t
\end{aligned}
$$

as we wanted to prove. 
Definition 2.11. We define the spaces $D_{p, \alpha}^{-}$and $R_{p, \alpha}^{-}$by the norms

$$
\|f\|_{D_{p, \alpha}^{-}}=\|f\|_{p}+\int_{1}^{\infty} \frac{\left(\int_{t}^{\infty} f^{* *}(s)^{p} d s\right)^{1 / p}}{t}\left(1+\log ^{+} t\right)^{\alpha-1} d t
$$

and

$$
\|f\|_{R_{p, \alpha}^{-}}=\sup _{t>0} \frac{\left(\int_{t}^{\infty} f^{* *}(s)^{p} d s\right)^{1 / p}}{\left(1+\log ^{+} \frac{1}{t}\right)^{\alpha}} .
$$

Also

$$
D_{p, \alpha, \text { rad }}^{-}=\left\{f \in D_{p, \alpha}^{-}: f \text { is radial }\right\} .
$$

With these definitions, Theorem 2.10 reads as follows:

TheOREM 2.12. Under the hypothesis of Theorem 2.10,

$$
T: D_{p_{1}, \alpha, \mathrm{rad}}^{-} \rightarrow R_{p_{1}, \alpha}^{-}
$$

is bounded.

As before, let us now make some comparison with classical spaces.

REMARK 2.13. 1. Let us see that Theorem 2.10 improves Theorem 2.8: first of all, by a discretization argument, it is easy to see that

$$
\left(\int_{t}^{\infty} f^{* *}(s)^{p_{1}} d s\right)^{1 / p_{1}} \lesssim \int_{t}^{\infty} f^{* *}(s) s^{1 / p_{1}-1} d s
$$

and hence

$$
\begin{aligned}
\|f\|_{D_{p_{1}, \alpha}^{-}} & =\|f\|_{p_{1}}+\int_{1}^{\infty} \frac{\left(\int_{t}^{\infty} f^{* *}(s)^{p_{1}} d s\right)^{1 / p_{1}}}{t}\left(1+\log ^{+} t\right)^{\alpha-1} d t \\
& \lesssim\|f\|_{p_{1}}+\int_{1}^{\infty} \frac{\int_{t}^{\infty} f^{* *}(s) s^{1 / p_{1}-1} d s}{t}\left(1+\log ^{+} t\right)^{\alpha-1} d t \\
& =\|f\|_{p_{1}}+\int_{1}^{\infty} f^{* *}(s) s^{1 / p_{1}-1} \int_{1}^{s} \frac{\left(1+\log ^{+} t\right)^{\alpha-1}}{t} d t \\
& \approx\|f\|_{p_{1}}+\int_{1}^{\infty} f^{* *}(s) s^{1 / p_{1}-1}\left(1+\log ^{+} s\right)^{\alpha} d s \\
& \lesssim \int_{0}^{\infty} f^{*}(u) \int_{\max (1, u)}^{\infty} s^{1 / p_{1}-2}\left(1+\log ^{+} s\right)^{\alpha} d s \\
& \approx \int_{0}^{\infty} f^{*}(u) u^{1 / p_{1}-1}\left(1+\log ^{+} u\right)^{\alpha} d u \\
& =\|f\|_{\Lambda^{1}\left(v_{0}\right)}
\end{aligned}
$$


with $v_{0}$ as in Theorem 2.8. Hence

$$
\Lambda^{1}\left(v_{0}\right) \subset D_{p_{1}, \alpha}^{-} .
$$

The inclusion is strict since taking $f$ such that $f^{*}(s)=s^{-1 / p_{1}}\left(1+\log ^{+} u\right)^{-\beta}$ with $\alpha+1 / p_{1}<\beta \leq 1+\alpha$ we obtain $\|f\|_{\Lambda^{1}\left(v_{0}\right)}=\infty$ and $\|f\|_{D_{p_{1}, \alpha}^{-}}<\infty$. Also taking $h^{*}(t)=t^{1 / p_{1}}$ one can imediately see that $R_{p_{1}, \alpha}^{-} \subset \Gamma^{1, \infty}\left(v_{1}\right)$ with $v_{1}$ as in Theorem 2.8 and the embedding is strict.

2. In particular, if $f \in L^{p_{1}}(\log 1 / L)^{\beta}$ with $\beta>\alpha p_{1}$, that is,

$$
\int_{\mathbb{R}^{n}}|f(x)|^{p_{1}}\left(1+\log ^{+} \frac{1}{|f(x)|}\right)^{\beta} d x<\infty
$$

then

$$
\begin{aligned}
& \int_{1}^{\infty} \frac{\left(\int_{t}^{\infty} f^{* *}(s)^{p_{1}} d s\right)^{1 / p_{1}}}{t}(\log t)^{\alpha-1} d t \\
& \quad \leq\left(\int_{0}^{\infty} f^{* *}(s)^{p_{1}}\left(1+\log ^{+} s\right)^{\beta} d s\right)^{1 / p_{1}} \int_{1}^{\infty} \frac{(\log t)^{\alpha-1}}{t(1+\log t)^{\beta / p_{1}}} d t \\
& \quad \lesssim\left(\int_{0}^{\infty} f^{* *}(s)^{p_{1}}\left(1+\log ^{+} s\right)^{\beta} d s\right)^{1 / p_{1}} \approx\left(\int_{0}^{\infty} f^{*}(s)^{p_{1}}\left(1+\log ^{+} s\right)^{\beta} d s\right)^{1 / p_{1}}
\end{aligned}
$$

where the last inequality follows since $\left(1+\log ^{+} s\right)^{\beta} \in B_{p_{1}}$ (see [3]). Finally, since $\sup _{s} f^{*}(s) s^{1 / p_{1}}<\infty$ we have

$$
\begin{aligned}
\left(\int_{0}^{\infty} f^{*}(s)^{p_{1}}\left(1+\log ^{+} s\right)^{\beta} d s\right)^{1 / p_{1}} & \lesssim\left(\int_{0}^{\infty} f^{*}(s)^{p_{1}}\left(1+\log \frac{1}{f^{*}(s)}\right)^{\beta} d s\right)^{1 / p_{1}} \\
& \approx\left(\int_{\mathbb{R}^{n}}|f(x)|^{p_{1}}\left(1+\log ^{+} \frac{1}{|f(x)|}\right)^{\beta} d x\right)^{1 / p_{1}}
\end{aligned}
$$

and therefore $L^{p_{1}}(\log 1 / L)^{\beta} \subset D_{p_{1}, \alpha}^{-}$.

\section{Applications}

3.1. Almost everywhere convergence of spherical partial Fourier integrals for radial functions in weighted spaces. In [25] one of the authors proved that if $f$ is a radial function belonging to $L^{p}\left(\mathbb{R}^{n}\right), 2 n /(n+1)<p<2 n /(n-1)$, then $S_{R} f(x)$ converges a.e. to $f(x)$ whenever $R$ tends to $\infty$, where

$$
S_{R} f(x)=\int_{B(0, R)} \hat{f}(\xi) e^{2 \pi i x \xi} d \xi
$$


is the spherical partial Fourier integral. To do this it was shown that, for radial functions $f$,

$$
\tilde{S} f(x)=\sup _{R}\left|S_{R} f(x)\right| \leq \frac{C(n)}{s^{(n-1) / 2}}(M+L+\tilde{H}+\tilde{C})(g)(s)
$$

where $s=|x|, g(r)=f(r) r^{(n-1) / 2} \chi_{(0, \infty)}(r), M$ is the Hardy-Littlewood maximal operator, $\tilde{H}$ is the maximal Hilbert transform, $\tilde{C}$ is the maximal Carleson operator defined by

$$
\tilde{C} f(x)=\sup _{y \in \mathbb{R}} \sup _{\varepsilon>0}\left|\int_{\varepsilon<|x-t|} \frac{e^{-i y t} f(t)}{x-t} d t\right|
$$

and $L$ is the Hilbert integral

$$
L f(s)=\int_{0}^{\infty} \frac{f(t)}{s+t} d t .
$$

Using (3.1) it is proved in [27] and [15] that

$$
\tilde{S}: L_{\mathrm{rad}}^{p_{j}, 1} \rightarrow L^{p_{j}, \infty}, \quad j=0,1,
$$

is bounded with

$$
p_{0}=\frac{2 n}{n+1} \quad \text { and } \quad p_{1}=\frac{2 n}{n-1} .
$$

From this the almost everywhere convergence of $S_{R} f(x)$ in $L_{\text {rad }}^{p_{j}, 1}$ follows.

Again (3.1) is used in [26] to prove that if $v$ is a radial weight such that $u(s)=v(s) s^{(n-1)(1-p / 2)} \in A_{p}$ then

$$
\|\tilde{S} f\|_{L^{p}(v)} \leq C_{v, p}\|f\|_{L_{\mathrm{rad}}^{p}(v)}
$$

but no information is given about the behaviour of the constant $C_{v, p}$.

In this section, we shall give an estimate of the constant $C_{w, p}$ for any radial weight $w$ on $\mathbb{R}^{n}$ provided $w_{0} \in A_{1}$, where $w_{0}(r)=w(|x|)$ for $|x|=$ $r>0$ and $w_{0}(r)=w_{0}(-r)$ for $r<0$. Recall $w_{0} \in A_{1}$ if

$$
M w_{0}(s) \leq C w_{0}(s) \quad \text { a.e. } s \in \mathbb{R},
$$

and $\left\|w_{0}\right\|_{A_{1}}$ is the infimum of all the above constants $C$.

When $w: \mathbb{R}^{n} \rightarrow \mathbb{R}^{+}$is a radial function such that $w_{0} \in A_{1}$, we shall write $w \in A_{1}(\mathbb{R})$ and $\|w\|_{A_{1}(\mathbb{R})}=\left\|w_{0}\right\|_{A_{1}}$.

With this notation we shall prove that, for every $p_{0}<p \leq 2$,

$$
C_{w, p} \lesssim\|w\|_{A_{1}(\mathbb{R})}\left(\frac{1}{p-p_{0}}\right)^{3}
$$

Then we can apply Theorem 2.6 and deduce the convergence almost everywhere of the spherical partial Fourier integrals for radial functions in 
$D_{p_{0}, 3, \text { rad }}^{+}(w)$, which is $D_{p_{0}, 3, \text { rad }}^{+}$with $\mu=w(x) d x$; similarly for $R_{p_{0}, 3}^{+}(w)$. Precisely, we shall prove the following:

TheOREM 3.1. If $w$ is a radial function in $\mathbb{R}^{n}$ such that $w \in A_{1}(\mathbb{R})$ then

$$
\tilde{S}: D_{p_{0}, 3, \operatorname{rad}}^{+}(w) \rightarrow R_{p_{0}, 3}^{+}(w)
$$

is bounded. Hence if $f \in D_{p_{0}, 3, \mathrm{rad}}^{+}(w)$, then

$$
S_{R} f(x) \rightarrow f(x)
$$

as $R \rightarrow \infty$ for almost every $x \in \mathbb{R}^{n}$. In particular, (3.3) holds for every radial function $f$ satisfying

$$
\int_{0}^{\infty} f_{w}^{*}(t) t^{1 / p_{0}}\left(1+\log ^{+} \frac{1}{t}\right)^{3} \frac{d t}{t}<\infty
$$

and every radial function in $L^{p_{0}}(\log L)^{\beta}(w)$ with $\beta>3 p_{0}$.

Proof. From (3.1) we have

$$
\|\tilde{S} f\|_{L^{p}(w)} \lesssim\|T g\|_{L^{p}\left(\mathbb{R}^{+} ; w(s) s^{(n-1)(1-p / 2)}\right)} \leq\|T g\|_{L^{p}(\mathbb{R} ; u)}
$$

where $u(s)=w(s)|s|^{(n-1)(1-p / 2)}$ and, for $s \in \mathbb{R}$,

$$
T g(s)=(M+L+\tilde{H}+\tilde{C})(g)(|s|) .
$$

To give an estimate of the constant $C(w, p)$ in the inequality

$$
\|T g\|_{L^{p}(u)} \leq C(w, p)\|g\|_{L^{p}(u)}
$$

we will go through the following steps:

SteP 1. If $-1<\alpha \leq 0$ and $v_{\alpha}(s)=|s|^{\alpha}$, with $s \in \mathbb{R}$, then

$$
\left\|v_{\alpha}\right\|_{A_{1}} \leq \frac{2}{1+\alpha}
$$

Then recalling that $u(s)=w(s)|s|^{(n-1)(1-p / 2)}$ and $w \in A_{1}(\mathbb{R})$, we shall prove that, for every $p_{0}<p \leq 2$, the following four steps hold:

STEP 2.

$$
\|u\|_{A_{p}} \lesssim\|w\|_{A_{1}(\mathbb{R})}\left(\frac{1}{p-p_{0}}\right)^{p-1}
$$

STEP 3.

$$
\|M g\|_{L^{p}(u)} \leq \frac{\|w\|_{A_{1}(\mathbb{R})}^{(n+1) /(n-1)}}{p-p_{0}}\|g\|_{L^{p}(u)} .
$$

STEP 4.

$$
\|L g\|_{L^{p}(u)} \leq \frac{\|w\|_{A_{1}(\mathbb{R})}^{(n+1) /(n-1)}}{p-p_{0}}\|g\|_{L^{p}(u)} .
$$


STEP 5.

$$
\|(\tilde{H}+\tilde{C}) g\|_{L^{p}(u)} \lesssim\|w\|_{A_{1}(\mathbb{R})}^{(n+1) /(n-1)}\left(\frac{1}{p-p_{0}}\right)^{3}\|g\|_{L^{p}(u)} .
$$

From Steps 3-5 it follows that

$$
\|\tilde{S} f\|_{L^{p}(w)} \lesssim\|w\|_{A_{1}(\mathbb{R})}^{(n+1) /(n-1)}\left(\frac{1}{p-p_{0}}\right)^{3}\|g\|_{L^{p}(u)} .
$$

Then the result follows by Theorem 2.4, since trivially $\|g\|_{L^{p}(u)} \approx\|f\|_{L^{p}(w)}$.

Proof of Step 1. Since $v_{\alpha}$ is even, so also is

$$
M\left(v_{\alpha}\right)(x)=\sup _{a<x<b} \frac{1}{b-a} \int_{a}^{b}|s|^{\alpha} d s
$$

and hence we can assume $x>0$. If $a>0$ then from $-1<\alpha<0$ it follows that $b^{\alpha}<x^{\alpha}<a^{\alpha}$ and

$$
\frac{1}{b-a} \int_{a}^{b}|s|^{\alpha} d s=\frac{1}{1+\alpha} \frac{b^{1+\alpha}-a^{1+\alpha}}{b-a} \leq \frac{1}{1+\alpha} \frac{x^{\alpha} b-x^{\alpha} a}{b-a}=\frac{x^{\alpha}}{1+\alpha} .
$$

If $a<0$ and $-a>b$ then

$$
\frac{1}{b-a} \int_{a}^{b}|s|^{\alpha} d s=\frac{1}{1+\alpha} \frac{b^{1+\alpha}+(-a)^{1+\alpha}}{b-a} \leq \frac{2}{1+\alpha} b^{\alpha} \leq \frac{2 x^{\alpha}}{1+\alpha} .
$$

If $a<0$ and $-a<b, 0<B=-a / b<1$ then

$$
\frac{1}{b-a} \int_{a}^{b}|s|^{\alpha} d s=\frac{1}{1+\alpha} \frac{b^{1+\alpha}+(-a)^{1+\alpha}}{b-a} \leq \frac{1}{1+\alpha} b^{\alpha} \frac{1+B^{1+\alpha}}{1+B} \leq \frac{2 x^{\alpha}}{1+\alpha} .
$$

Therefore

$$
M\left(v_{\alpha}\right)(x) \leq \frac{2 x^{\alpha}}{1+\alpha} .
$$

Proof of Step 2. This result follows from the fact (see [16]) that if $w_{0}, w_{1} \in A_{1}$, then $w_{0} w_{1}^{1-p} \in A_{p}$ and

$$
\left\|w_{0} w_{1}^{1-p}\right\|_{A_{p}} \leq\left\|w_{0}\right\|_{A_{1}}\left\|w_{1}\right\|_{A_{1}}^{p-1} .
$$

Hence writing

$$
w_{0}=w \quad \text { and } \quad w_{1}(s)=|s|^{\frac{(n-1)(1-p / 2)}{1-p}}
$$

we see that $u=w_{0} w_{1}^{1-p}$ and thus by Step 1 ,

$$
\begin{aligned}
\|u\|_{A_{p}} & \leq\|w\|_{A_{1}}\left\|w_{1}\right\|_{A_{1}}^{p-1} \lesssim\|w\|_{A_{1}}\left(\frac{1}{1+\frac{(n-1)(1-p / 2)}{1-p}}\right)^{p-1} \\
& \lesssim\|w\|_{A_{1}(\mathbb{R})}\left(\frac{1}{p-p_{0}}\right)^{p-1} .
\end{aligned}
$$


Proof of Step 3. By [6] (see also [23]) we know that

$$
\|M g\|_{L^{p}(u)} \leq\|u\|_{A_{p}}^{1 /(p-1)}\|g\|_{L^{p}(u)},
$$

and hence the result follows by Step 2 since $1 /(p-1) \leq(n+1) /(n-1)$.

Proof of Step 4. First we observe that

$$
\begin{aligned}
L g(|s|) & =\int_{0}^{\infty} \frac{g(t)}{t+|s|} d t \leq \frac{1}{|s|} \int_{0}^{2|s|} g(t) d t+\int_{|s|}^{\infty} \frac{g(t)}{t} d t \\
& \lesssim M g(|s|)+\int_{|s|}^{\infty} \frac{g(t)}{t} d t=M g(|s|)+R g(s) .
\end{aligned}
$$

For the first term, we can apply Step 3, and for the second one we shall proceed by duality:

$$
\begin{aligned}
\|R g\|_{L^{p}(u)} & =\sup _{\|h\|_{L^{p^{\prime}}\left(u-p^{\prime} / p\right)} \leq 1}\left|\int\left(\int_{|s|}^{\infty} \frac{g(t)}{t} d t\right) h(s) d s\right| \\
& =\sup _{\|h\|_{L^{p^{\prime}\left(u-p^{\prime} / p\right)}} \leq 1}\left|\int g(t)\left(\frac{1}{t} \int_{|s| \leq t} h(s) d s\right) d t\right| \\
& \lesssim \sup _{\|h\|_{L^{p^{\prime}}\left(u-p^{\prime} / p\right)} \leq 1} \int|g(t)| M h(t) d t \leq\|g\|_{L^{p}(u)}\|M h\|_{L^{p^{\prime}\left(u^{-p^{\prime}} / p\right)}} .
\end{aligned}
$$

Using (3.5), we obtain

$$
\|M h\|_{L^{p^{\prime}}\left(u^{\left.-p^{\prime} / p\right)}\right.} \leq\left\|u^{-p^{\prime} / p}\right\|_{A_{p^{\prime}}}^{1 /\left(p^{\prime}-1\right)}\|h\|_{L^{p^{\prime}\left(u^{-p^{\prime} / p}\right)}}
$$

and hence

$$
\|R g\|_{L^{p}(u)} \leq\left\|u^{-p^{\prime} / p}\right\|_{A_{p^{\prime}}}^{1 /\left(p^{\prime}-1\right)}\|g\|_{L^{p}(u)} .
$$

Since (see [16]) $u^{-p^{\prime} / p} \in A_{p^{\prime}}$ if and only if $u \in A_{p}$ and, in fact,

$$
\left\|u^{-p^{\prime} / p}\right\|_{A_{p^{\prime}}}=\|u\|_{A_{p}}^{p^{\prime}-1}
$$

applying Step 2 for $p \leq 2$, we obtain

$$
\begin{aligned}
\|R g\|_{L^{p}(u)} & \leq\|u\|_{A_{p}}\|g\|_{L^{p}(u)} \lesssim\|w\|_{A_{1}}\left(\frac{1}{p-p_{0}}\right)^{p-1}\|g\|_{L^{p}(u)} \\
& \leq \frac{\|w\|_{A_{1}}}{p-p_{0}}\|g\|_{L^{p}(u)} .
\end{aligned}
$$

Proof of Step 5. For this step we refer to [21] where the following goodlambda inequality is proved, for a decomposition of $\{\tilde{C} f(x)>\lambda\}$ into pairwise disjoint intervals $\left(I_{j}\right)_{j}$ :

$$
\left|\left\{x \in I_{j}: \tilde{C} f(x)>3 \lambda, M_{r} f(x) \leq \gamma \lambda\right\}\right| \leq C_{r} \gamma^{r}\left|I_{j}\right|,
$$


where $M_{r} f(x)=\left(M\left(|f|^{r}\right)(x)\right)^{1 / r}, 1<r<p$ and $\gamma>0$ is small enough. We are interested in the behaviour of $C_{r}$ as $r \rightarrow 1$. The proof shows that $C_{r} \lesssim 1 /(r-1)^{2}$ since $\|\tilde{C}\|_{L^{r} \rightarrow L^{r, \infty}} \lesssim 1 /(r-1)^{2}$ ([8], [20]). Hence, for $r>1$,

$$
\left|\left\{x \in I_{j}: \tilde{C} f(x)>3 \lambda, M_{r} f(x) \leq \gamma \lambda\right\}\right| \lesssim \frac{\gamma^{r}}{(r-1)^{2}}\left|I_{j}\right| .
$$

Now we observe that even though our weight $u$ depends on $p$, by using Step 1 and (3.4), it follows that, for every $2 n /(n+1)<p \leq 2$,

$$
\begin{aligned}
\|u\|_{A_{2}} & \leq\|w\|_{A_{1}}\left\||s|^{(n-1)(p / 2-1)}\right\|_{A_{1}} \lesssim\|w\|_{A_{1}} \frac{1}{1+(n-1)(p / 2-1)} \\
& \leq \frac{n+1}{2}\|w\|_{A_{1}} .
\end{aligned}
$$

Hence the norm of $u$ as a weight in $A_{\infty}$ is uniformly bounded on $p$. Since $u \in A_{\infty}$ implies that there exist $C$ and $\delta$ depending on $\|u\|_{A_{\infty}}$ such that, for every subset $S$ of an interval $I$,

we obtain

$$
\frac{u(S)}{u(I)} \leq C\left(\frac{|S|}{|I|}\right)^{\delta}
$$

$$
u\left(\left\{x \in I_{j}: \tilde{C} f(x)>3 \lambda, M_{r} f(x) \leq \gamma \lambda\right\}\right) \lesssim\left(\frac{\gamma^{r}}{(r-1)^{2}}\right)^{\delta} u\left(I_{j}\right) .
$$

Summing over $j$ we have

$$
u\left(\left\{x: \tilde{C} f(x)>3 \lambda, M_{r} f(x) \leq \gamma \lambda\right\}\right) \lesssim\left(\frac{\gamma^{r}}{(r-1)^{2}}\right)^{\delta} u(\{\tilde{C} f(x)>\lambda\}) .
$$

From this inequality and using standard techniques we deduce that, for $r>1$,

and thus

$$
\|\tilde{C} g\|_{L^{p}(u)} \lesssim \frac{1}{(r-1)^{2}}\left\|M_{r} g\right\|_{L^{p}(u)}
$$

$$
\|\tilde{C} g\|_{L^{p}(u)} \lesssim \frac{1}{(r-1)^{2}}\|u\|_{A_{p / r}}^{\frac{r}{p / r-1}}\|g\|_{L^{p}(u)} \lesssim \frac{1}{(r-1)^{2}} \frac{\|w\|_{A_{1}}^{\frac{r}{p / r-1}}}{1+\frac{(n-1)(1-p / 2)}{1-p / r}}\|g\|_{L^{p}(u)} .
$$

Let us choose $r$ such that

$$
1-\frac{1}{r}=c\left(p-\frac{2 n}{n+1}\right),
$$

with $c$ small enough. Then it is easy to see that if $p$ is near $2 n /(n+1)$, then $1<r<p$ and

$$
\frac{1}{(r-1)^{2}} \frac{1}{1+\frac{(n-1)(1-p / 2)}{1-p / r}} \approx\left(\frac{1}{p-p_{0}}\right)^{3},
$$

and the result follows.

The particular cases are consequences of Remark 2.7. 
At the right endpoint $p_{1}$, it is not true that $u(s)=w(s)|s|^{(n-1)(1-p / 2)} \in$ $A_{p}$ with $p$ near $p_{1}$ for every $w \in A_{1}$ and thus we have to impose another condition on $w$ :

Theorem 3.2. If $w(s)|s|^{-2 n /(n-1)} \in A_{1}(\mathbb{R})$ then

$$
\tilde{S}: D_{p_{1}, 3, \operatorname{rad}}^{+}(w) \rightarrow R_{p_{1}, 3}^{+}(w)
$$

is bounded. Hence if $f \in D_{p_{1}, 3, \mathrm{rad}}^{+}(w)$ then $S_{R} f(x) \rightarrow f(x)$ as $R \rightarrow \infty$ for almost every $x \in \mathbb{R}^{n}$. In particular, the almost everywhere convergence holds for every radial function satisfying

$$
\int_{0}^{\infty} f^{*}(u) u^{1 / p_{1}-1}\left(1+\log ^{+}(1 / u)\right)^{3} d u<\infty
$$

or $f \in L^{p_{1}}(\log L)^{\beta}$ with $\beta>3 p_{1}$.

Proof. The proof follows the same steps as that of Theorem 3.1 as soon as we prove that $u \in A_{p}$. We shall use the fact that $u \in A_{p}$ if and only if $u^{1-p^{\prime}} \in A_{p^{\prime}}$ and that $\|u\|_{A_{p}}=\left\|u^{1-p^{\prime}}\right\|_{A_{p^{\prime}}}^{1 /\left(p^{\prime}-1\right)}$ and write

$$
u(s)^{1-p^{\prime}}=\left(w(s)|s|^{-2 n /(n-1)}\right)^{1-p^{\prime}}|s|^{((n-1)(1-p / 2)+2 n /(n-1))\left(1-p^{\prime}\right)} .
$$

To see that $u^{1-p^{\prime}} \in A_{p^{\prime}}$ it is enough to check $|s|^{((n-1)(1-p / 2)+2 n /(n-1))\left(1-p^{\prime}\right)} \in$ $A_{1}$ and hence we need

$$
-1<\left((n-1)\left(1-\frac{p}{2}\right)+\frac{2 n}{n-1}\right)\left(1-p^{\prime}\right) \leq 0,
$$

which trivially holds for $p$ near $p_{1}, p>p_{1}$, and by Step 1 in the proof of Theorem 3.1 and (3.4),

$$
\begin{aligned}
& \|u\|_{A_{p}}=\left\|u^{1-p^{\prime}}\right\|_{A_{p^{\prime}}}^{1 /\left(p^{\prime}-1\right)} \\
& \quad \lesssim\left(\left\|w(s)|s|^{-2 n /(n-1)}\right\|_{1}^{p^{\prime}-1} \frac{1}{1+\left(1-p^{\prime}\right)\left((n-1)(1-p / 2)+\frac{2 n}{n-1}\right)}\right)^{1 /\left(p^{\prime}-1\right)} \\
& \quad \lesssim\left\|w(s)|s|^{-2 n /(n-1)}\right\|_{1}\left(\frac{1}{p-1-(n-1)(1-p / 2)-\frac{2 n}{n-1}}\right)^{p-1} \\
& \quad \lesssim\left\|w(s)|s|^{-2 n /(n-1)}\right\|_{1}\left(\frac{1}{p-\frac{2 n}{n-1}}\right)^{p-1} .
\end{aligned}
$$

Following now the same steps as in the proof of Theorem 3.1, it remains only to prove that $u \in A_{\infty}$; but this follows easily since, in fact, for $n>1$, $u \in A_{3}$ using again (3.4) and Step 1.

The particular cases follow from Remark 2.13. 
3.2. Endpoint estimates for maximal Bochner-Riesz type operators acting on radial functions. In [19] the following theorem for maximal BochnerRiesz type operators was proved:

Theorem 3.3. Let $T f(x)=\sup _{t>0}\left|\left(K_{t} * f\right)(x)\right|$, where $K: \mathbb{R}^{n} \rightarrow \mathbb{C}$ is a radial, bounded measurable function, and let $0<\delta<(n-1) / 2$. Suppose that $K(x)=a(|x|) e^{i \varphi(|x|)}$ for $|x|>\varrho$, where

$$
\left|\frac{d^{m}}{d s^{m}} a(s)\right| \leq c s^{-((n+1) / 2+\delta+m)}, \quad\left|\frac{d^{m}}{d s^{m}}\left(\varphi^{\prime}(s)\right)^{-1}\right| \leq c s^{-m},
$$

for $s>\varrho$ and $m=0, \ldots,[n / 2]$. Then $T$ is bounded on $L_{\mathrm{rad}}^{p}\left(\mathbb{R}^{n}\right)$ for every $2 n /(n+1+2 \delta)<p<2 n /(n-1-2 \delta)$.

REMARK 3.4. In [19] it is also proved that, for $n$ even, $T$ is of weak type on $L_{\mathrm{rad}}^{p}\left(\mathbb{R}^{n}\right)$ for $p=2 n /(n+1+2 \delta)$ and restricted weak type for $p=2 n /(n-1-2 \delta)$. If $n$ is odd the same is proved under the additional assumption that (3.6) also holds for $m=(n+1) / 2$.

Set

$$
p_{0}(\delta)=\frac{2 n}{n+1+2 \delta} \quad \text { and } \quad p_{1}(\delta)=\frac{2 n}{n-1-2 \delta} .
$$

Claim. If $T$ satisfies the hypothesis of Theorem 3.3 then

$$
\|T f\|_{p, \infty} \lesssim \frac{1}{p-p_{0}(\delta)}\left(\frac{1}{p_{1}(\delta)-p}\right)^{(3 n+1+2 \delta) / 2 n}\|f\|_{L_{\mathrm{rad}}^{p}} .
$$

Proof. This follows by carefully checking Epperson's calculations. First of all, it turns out that the constant $c$ in the statement of Lemma 1.4 in [19] satisfies

$$
c=c(\eta) \lesssim 1 / \eta .
$$

Indeed, the computations involve $\int_{k_{1}}^{\pi / 2} \theta^{-(1+\eta)} d \eta$ and $\int_{\pi / 2}^{k_{2}}(\pi-\theta)^{-(1+\eta)} d \eta$ (Case 4, p. 114), which are both bounded by $C / \eta$. The other cases give better constants.

Now, the proof of Theorem 1.1 in [19] shows that

$$
\|T f\|_{L^{p, \infty}} \lesssim\left(\max _{i=1, \ldots, 6} a_{i}\right)\|f\|_{p}
$$

where the $a_{i}$ are given by

$$
\int_{C_{\lambda}^{i}} s^{n-1} d s \leq a_{i}^{p}\left(\frac{\|f\|_{p}}{\lambda}\right)^{p} .
$$

Following Epperson's calculations we see that for $i=1,3,5,6$,

$$
a_{i} \lesssim c(\eta)
$$


For $i=2,4$,

$$
a_{i} \lesssim \frac{c(\eta)}{\eta^{1 / p^{\prime}}}
$$

if $p$ is close to $p_{1}(\delta)$, and $a_{i} \lesssim c(\eta)$ if $p$ is close to $p_{0}(\delta)$.

From this our claim follows. Indeed, if $p<p_{1}(\delta)$, we choose $\eta>0$ such that $p_{1}(\bar{\delta})<p$ with $\bar{\delta}=\delta-\eta$ and so we have

$$
p_{1}(\delta)-p_{1}(\bar{\delta}) \lesssim \eta
$$

and consequently

$$
\frac{1}{\eta} \lesssim \frac{1}{p_{1}(\delta)-p}
$$

Hence, for $p$ close to $p_{1}(\delta)$, we obtain

$$
\|T f\|_{L^{p, \infty}} \lesssim\left(\frac{1}{p_{1}(\delta)-p}\right)^{1+1 / p^{\prime}}\|f\|_{L_{\mathrm{rad}}^{p}}
$$

which implies

$$
\|T f\|_{L^{p, \infty}} \lesssim\left(\frac{1}{p_{1}(\delta)-p}\right)^{(3 n+1+2 \delta) / 2 n}\|f\|_{L_{\text {rad }}^{p}},
$$

and similarly, for $p$ close to $p_{0}(\delta)$,

$$
\|T f\|_{L^{p, \infty}} \lesssim \frac{1}{p-p_{0}(\delta)}\|f\|_{L_{\mathrm{rad}}^{p}}
$$

TheOREM 3.5. Under the hypothesis of the previous theorem,

$$
T: \Lambda_{\mathrm{rad}}^{1}\left(v_{0}\right) \rightarrow \Gamma^{1, \infty}\left(v_{1}\right)
$$

is bounded with

(i) $v_{0}(t)=t^{1 / p_{0}(\delta)-1}\left(1+\log ^{+} \frac{1}{t}\right) \quad$ and $\quad v_{1}(t)=t^{1 / p_{0}(\delta)-1}\left(1+\log ^{+} t\right)^{-1}$ and also

(ii) $v_{0}(t)=t^{1 / p_{1}(\delta)-1}\left(1+\log ^{+} t\right)^{\alpha} \quad$ and $\quad v_{1}(t)=t^{1 / p_{1}(\delta)-1}\left(1+\log ^{+} \frac{1}{t}\right)^{-\alpha}$ with $\alpha=(3 n+1+2 \delta) / 2 n$.

Proof. (i) Near $p_{0}(\delta)$ we have

$$
\|T f\|_{p, \infty} \lesssim \frac{1}{p-p_{0}(\delta)}\|f\|_{p}
$$

Now, from the proof of Theorem 2.1, one can easily see that, in fact, the only condition on $T$ that we use is that

$$
\sup _{t>0}(T f)^{* *}(t) t^{1 / p} \lesssim \frac{1}{p-p_{0}}\|f\|_{p},
$$

and this condition follows immediately from (3.7) since $p>p_{\delta}>1$. 
(ii) Near $p_{1}(\delta)$, we have

$$
\|T f\|_{p, \infty} \lesssim\left(\frac{1}{p_{1}(\delta)-p}\right)^{(3 n+1+2 \delta) / 2 n}\|f\|_{p},
$$

but from the proof of Theorem 2.8, one can easily see that, in fact, the only condition on $T$ that we use is that

$$
\sup _{t>0}(T f)^{* *}(t) t^{1 / p} \lesssim\left(\frac{1}{p_{1}(\delta)-p}\right)^{(3 n+1+2 \delta) / 2 n}\|f\|_{p},
$$

and this condition follows immediately from (3.8).

Acknowledgements. The first author was supported by MTM200760500 and by CURE 2005SGR00556.

\section{References}

[1] K. Andersen, Weighted inequalities for the Stieltjes transform and the maximal spherical partial sum operator on radial functions, Proc. Roy. Soc. Edinburgh Sect. A 125 (1995), 195-204.

[2] N. Y. Antonov, Convergence of Fourier series, East J. Approx. 2 (1996), 187-196.

[3] M. A. Ariño and B. Muckenhoupt, Maximal functions on classical Lorentz spaces and Hardy's inequality with weights for nonincreasing functions, Trans. Amer. Math. Soc. 320 (1990), 727-735.

[4] C. Bennett and R. Sharpley, Interpolation of Operators, Pure Appl. Math. 129, Academic Press, Boston, 1988.

[5] Yu. A. Brudnyi and N. Ya. Krugljak, Interpolation Functors and Interpolation Spaces, North-Holland, 1991.

[6] S. M. Buckley, Estimates for operator norms on weighted spaces and reverse Jensen inequalities, Trans. Amer. Math. Soc. 340 (1993), 253-272.

[7] A. Carbery, E. Hernández and F. Soria, The behaviour on radial functions of maximal operators along arbitrary directions and the Kakeya maximal operator, Tohoku Math. J. 41 (1989), 647-656.

[8] L. Carleson, On convergence and growth of partial sums of Fourier series, Acta Math. 116 (1966), 135-157.

[9] M. Carro, From restricted weak type to strong type estimates, J. London Math. Soc. 70 (2004), 750-762.

[10] M. Carro and J. Martín, Endpoint estimates from restricted rearrangement inequalities, Rev. Mat. Iberoamer. 20 (2004), 131-150.

[11] —, - Extrapolation theory for the real interpolation method, Collect. Math. 53 (2002), 165-186.

[12] M. Carro, L. Pick, J. Soria and V. D. Stepanov, On embeddings between classical Lorentz spaces, Math. Inequal. Appl. 4 (2001), 397-428.

[13] M. Carro and J. Soria, Weighted Lorentz spaces and the Hardy operator, J. Funct. Anal. 112 (1993), 480-494.

[14] S. Chanillo and B. Muckenhoupt, Weak type estimates for Bochner-Riesz spherical summation multipliers, Trans. Amer. Math. Soc. 294 (1986), 693-703. 
[15] L. Colzani, A. Crespi, G. Travaglini and M. Vignati, Equiconvergence theorems for Fourier-Bessel expansions with applications to the harmonic analysis of radial functions in Euclidean and non-Euclidean spaces, Trans. Amer. Math. Soc. 338 (1993), 43-55.

[16] J. Duoandikoetxea, Fourier Analysis, Grad. Stud. in Math. 29, Amer. Math. Soc., Providence, RI, 2001.

[17] J. Duoandikoetxea and V. Naibo, The universal maximal operator on special classes of functions, Indiana Univ. Math. J. 54 (2005), 1351-1369.

[18] J. Duoandikoetxea and A. Vargas, Directional operators and radial functions on the plane, Ark. Mat. 33 (1995), 281-291.

[19] J. Epperson, Some estimates for radial Fourier multiplier operators with slowly decaying kernels, Math. Nachr. 191 (1998), 109-121.

[20] R. Hunt, On the convergence of Fourier series, in: Orthogonal Expansions and Their Continuous Analogues (Edwardsville, IL, 1967), Southern Illinois Univ. Press, 1968, 235-255.

[21] R. Hunt and W. Young, A weighted norm inequality for Fourier series, Bull. Amer. Math. Soc. 80 (1974), 274-277.

[22] Y. Kanjin, Convergence and divergence almost everywhere of spherical means for radial functions, Proc. Amer. math. Soc. 103 (1988), 1063-1069.

[23] A. Lerner, An elementary approach to several results on the Hardy-Littlewood maximal operator, personal communication.

[24] B. Muckenhoupt, Weighted norm inequalities for the Hardy maximal function, Trans. Amer. Math. Soc. 165 (1972), 207-226.

[25] E. Prestini, Almost everywhere convergence of the spherical partial sums for radial functions, Monatsh. Math. 105 (1988), 207-216.

[26] E. Romera, Weighted bounds for the Carleson maximal operator in $R^{n}$, Rend. Circ. Mat. Palermo (2) 43 (1994), 98-106.

[27] E. Romera and F. Soria, Endpoint estimates for the maximal operator associated to spherical partial sums on radial functions, Monatsh. Math. 105 (1988), 207-216.

[28] A. Seeger, T. Tao and J. Wright, Endpoint mapping properties of spherical maximal operators, J. Inst. Math. Jussieu 2 (2003), 109-144.

[29] A. Seeger, S. Wainger and J. Wright, Spherical maximal operators on radial functions, Math. Nachr. 187 (1997), 241-265.

[30] P. Sjölin and F. Soria, Remarks on a theorem by N. Y. Antonov, Studia Math. 158 (2003), 79-97.

[31] S. Yano, Notes on Fourier analysis. XXIX. An extrapolation theorem, J. Math. Soc. Japan 3 (1951), 296-305.

Departament de Matemàtica Aplicada i Anàlisi

Universitat de Barcelona

08071 Barcelona, Spain

E-mail: carro@ub.edu
Dipartimento di Matematica Università di Roma "Tor Vergata" 00133 Rome, Italy E-mail: prestini@mat.uniroma2.it

Received May 24, 2008

Revised version October 28, 2008 